\title{
Surge of subacute sclerosing panencephalitis
}

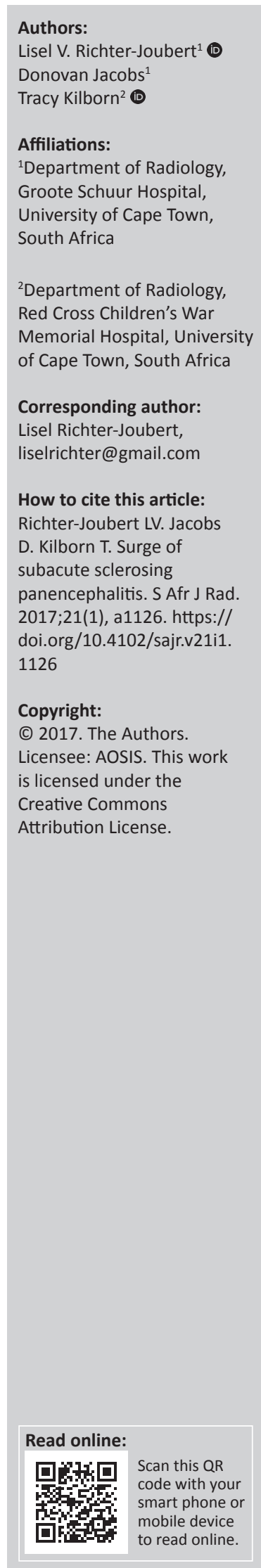

Subacute sclerosing panencephalitis (SSPE) is a poorly understood complication of measles infection, presenting in up to 1 in 2500 infected children, resulting in devastating neurocognitive outcomes and a high mortality rate. The onset is insidious, with patients presenting with seizures and progressive cognitive decline around 6-10 years after measles infection. The diagnosis is frequently delayed due to lack of clinical suspicion, but can be confirmed by typical electroencephalogram (EEG) findings and positive cerebrospinal fluid (CSF) serology. Although imaging is not diagnostic, the vigilant radiologist has a unique opportunity to direct decisionmaking by identifying the correct clinical context and supportive MRI findings. Five years after the measles outbreak of 2009-2011, a rise in the incidence of SSPE can be expected in South Africa, as has been experienced at our institution. We present a series of seven confirmed SSPE patients that presented to Red Cross War Memorial Children's Hospital between 2014 and 2016 and describe the clinical and radiological features to raise awareness of the condition amongst radiologist and clinicians. Note: A selection of conference abstracts: RSSA/SASPI Paediatric Imaging Congress, 03-06 November 2016, Spier Estate, Stellenbosch,
South Africa. Faculty collaborators: Professor Kassa Darge (Body Imaging, University of Pennsylvania, Philadelphia, USA), Professor South Africa. Faculty collaborators: Professor Kassa Darge (Body Imaging, University of Pennsylvania, Philadelphia, USA), Professor
Edward Lee (Thoracic Imaging, Harvard University, USA), Professor Beverley Newman (Cardiac Imaging, Stanford University, California, USA), Professor Kimberly Applegate (Image Gently and Body Imaging, Emory University, Atlanta, USA) and Professor Savvas Andronikou (Thoracic Imaging, University of Bristol, UK) supported by South African Paediatric Radiologists, co-ordinated by Dr Jaishree Naidoo, President of the African Society of Paediatric Imaging and Head of Division of Paediatric Radiology, Charlotte Maxeke Johannesburg Academic Hospital. 